\title{
A monotone projection algorithm for fixed points of nonlinear operators
}

\author{
Changqun $\mathrm{Wu}^{1 *}$ and Lijuan Sun ${ }^{2}$
}

${ }^{\text {"Correspondence: }}$

houbuyong@yeah.net

'School of Business and

Administration, Henan University,

Kaifeng, 475000, China

Full list of author information is

available at the end of the article

\begin{abstract}
In this paper, a monotone projection algorithm is investigated for equilibrium and fixed point problems. Strong convergence theorems for common solutions of the two problems are established in the framework of reflexive Banach spaces.
\end{abstract}

MSC: 47H09; 47J25; 90C33

Keywords: asymptotically quasi- $\phi$-nonexpansive mapping; generalized asymptotically quasi- $\phi$-nonexpansive mapping; bifunction; equilibrium problem; fixed point

\section{Introduction and preliminaries}

Let $E$ be a real Banach space with the dual $E^{*}$. Recall that the normalized duality mapping $J$ from $E$ to $2^{E^{*}}$ is defined by

$$
J x=\left\{f^{*} \in E^{*}:\left\langle x, f^{*}\right\rangle=\|x\|^{2}=\left\|f^{*}\right\|^{2}\right\},
$$

where $\langle\cdot, \cdot\rangle$ denotes the generalized duality pairing. Let $B_{E}=\{x \in E:\|x\|=1\}$ be the unit ball of $E$. Recall that $E$ is said to be smooth iff $\lim _{t \rightarrow 0} \frac{\|x+t y\|-\|x\|}{t}$ exists for each $x, y \in B_{E}$. It is also said to be uniformly smooth iff the above limit is attained uniformly for $x, y \in B_{E}$. $E$ is said to be strictly convex iff $\left\|\frac{x+y}{2}\right\|<1$ for all $x, y \in E$ with $\|x\|=\|y\|=1$ and $x \neq y$. It is said to be uniformly convex iff $\lim _{n \rightarrow \infty}\left\|x_{n}-y_{n}\right\|=0$ for any two sequences $\left\{x_{n}\right\}$ and $\left\{y_{n}\right\}$ in $E$ such that $\left\|x_{n}\right\|=\left\|y_{n}\right\|=1$ and $\lim _{n \rightarrow \infty}\left\|\frac{x_{n}+y_{n}}{2}\right\|=1$. It is well known that $E$ is uniformly smooth if and only if $E^{*}$ is uniformly convex. In what follows, we use $\rightarrow$ and $\rightarrow$ to stand for weak and strong convergence, respectively. Recall that $E$ enjoys the Kadec-Klee property iff for any sequence $\left\{x_{n}\right\} \subset E$, and $x \in E$ with $x_{n} \rightarrow x$, and $\left\|x_{n}\right\| \rightarrow\|x\|$, then $\left\|x_{n}-x\right\| \rightarrow 0$ as $n \rightarrow \infty$. It is well known that if $E$ is a uniformly convex Banach space, then $E$ enjoys the Kadec-Klee property. Let $E$ be a smooth Banach space. Let us consider the functional defined by

$$
\phi(x, y)=\|x\|^{2}-2\langle x, J y\rangle+\|y\|^{2}, \quad \forall x, y \in E .
$$

Recently, Alber [1] introduced a generalized projection operator $\Pi_{C}$ in a Banach space $E$ which is an analogue of the metric projection $P_{C}$ in Hilbert spaces. Recall that the generalized projection $\Pi_{C}: E \rightarrow C$ is a map that assigns to an arbitrary point $x \in E$ the minimum point of the functional $\phi(x, y)$, that is, $\Pi_{C} x=\bar{x}$, where $\bar{x}$ is the solution to the minimization problem $\phi(\bar{x}, x)=\min _{y \in C} \phi(y, x)$. Existence and uniqueness of the operator $\Pi_{C}$ follows 
from the properties of the functional $\phi(x, y)$ and strict monotonicity of the mapping $J$. If $E$ is a reflexive, strictly convex and smooth Banach space, then $\phi(x, y)=0$ if and only if $x=y$. In Hilbert spaces, $\Pi_{C}=P_{C}$. It is obvious from the definition of function $\phi$ that $(\|x\|-\|y\|)^{2} \leq \phi(x, y) \leq(\|y\|+\|x\|)^{2}, \forall x, y \in E$.

Let $\mathbb{R}$ be the set of real numbers. Let $F$ be a bifunction from $C \times C$ to $\mathbb{R}$, where $\mathbb{R}$ denotes the set of real numbers. Let $\varphi: C \rightarrow \mathbb{R}$ be a real-valued function and $A: C \rightarrow E^{*}$ be a mapping. The so-called generalized mixed equilibrium problem is to find $p \in C$ such that

$$
F(p, y)+\langle A p, y-p\rangle+\varphi(y)-\varphi(p) \geq 0, \quad \forall y \in C .
$$

We use $\operatorname{GMEP}(F, A, \varphi)$ to denote the solution set of the equilibrium problem. That is,

$$
\operatorname{GMEP}(F, A, \varphi):=\{p \in C: F(p, y)+\langle A p, y-p\rangle+\varphi(y)-\varphi(z) \geq 0, \forall y \in C\} .
$$

Next, we give some special cases:

If $A=0$, then problem (1.1) is equivalent to finding $p \in C$ such that

$$
F(p, y)+\varphi(y)-\varphi(z) \geq 0, \quad \forall y \in C,
$$

which is called the mixed equilibrium problem.

If $F=0$, then problem (1.1) is equivalent to finding $p \in C$ such that

$$
\langle A p, y-p\rangle+\varphi(y)-\varphi(z) \geq 0, \quad \forall y \in C,
$$

which is called the mixed variational inequality of Browder type.

If $\varphi=0$, then problem (1.1) is equivalent to finding $p \in C$ such that

$$
F(p, y)+\langle A p, y-p\rangle \geq 0, \quad \forall y \in C,
$$

which is called the generalized equilibrium problem.

If $A=0$ and $\varphi=0$, then problem (1.1) is equivalent to finding $p \in C$ such that

$$
F(p, y) \geq 0, \quad \forall y \in C,
$$

which is called the equilibrium problem.

For solving the above problem, let us assume that the bifunction $F: C \times C \rightarrow \mathbb{R}$ satisfies the following conditions:

(A1) $F(x, x)=0, \forall x \in C$;

(A2) $F$ is monotone, i.e., $F(x, y)+F(y, x) \leq 0, \forall x, y \in C$;

(A3)

$$
\limsup _{t \downarrow 0} F(t z+(1-t) x, y) \leq F(x, y), \quad \forall x, y, z \in C ;
$$

(A4) for each $x \in C, y \mapsto F(x, y)$ is convex and weakly lower semi-continuous. 
Iterative algorithms have emerged as an effective and powerful tool for studying a wide class of problems which arise in economics, finance, image reconstruction, ecology, transportation, network, elasticity and optimization; see [2-27] and the references therein. The computation of solutions of nonlinear operator equations (inequalities) is important in the study of many real world problems. Recently, the study of the convergence of various iterative algorithms for solving various nonlinear mathematical models forms the major part of numerical mathematics.

Let $C$ be a nonempty subset of $E$, and let $T: C \rightarrow C$ be a mapping. In this paper, we use $F(T)$ to stand for the fixed point set of $T$. Recall that $T$ is said to be asymptotically regular on $C$ iff for any bounded subset $K$ of $C, \limsup _{n \rightarrow \infty}\left\{\left\|T^{n+1} x-T^{n} x\right\|: x \in K\right\}=0$. Recall that $T$ is said to be closed iff for any sequence $\left\{x_{n}\right\} \subset C$ such that $\lim _{n \rightarrow \infty} x_{n}=x_{0}$ and $\lim _{n \rightarrow \infty} T x_{n}=y_{0}$, then $T x_{0}=y_{0}$. Recall that a point $p$ in $C$ is said to be an asymptotic fixed point of $T$ iff $C$ contains a sequence $\left\{x_{n}\right\}$ which converges weakly to $p$ such that $\lim _{n \rightarrow \infty}\left\|x_{n}-T x_{n}\right\|=0$. The set of asymptotic fixed points of $T$ will be denoted by $\widetilde{F}(T)$. $T$ is said to be relatively nonexpansive iff $\widetilde{F}(T)=F(T) \neq \emptyset$ and

$$
\phi(p, T x) \leq \phi(p, x), \quad \forall x \in C, \forall p \in F(T) .
$$

$T$ is said to be relatively asymptotically nonexpansive iff $\widetilde{F}(T)=F(T) \neq \emptyset$ and

$$
\phi\left(p, T^{n} x\right) \leq\left(1+\mu_{n}\right) \phi(p, x), \quad \forall x \in C, \forall p \in F(T), \forall n \geq 1,
$$

where $\left\{\mu_{n}\right\} \subset[0, \infty)$ is a sequence such that $\mu_{n} \rightarrow 0$ as $n \rightarrow \infty$.

Recall that $T$ is said to be quasi- $\phi$-nonexpansive iff $F(T) \neq \emptyset$ and

$$
\phi(p, T x) \leq \phi(p, x), \quad \forall x \in C, \forall p \in F(T) .
$$

Recall that $T$ is said to be asymptotically quasi- $\phi$-nonexpansive iff there exists a sequence $\left\{\mu_{n}\right\} \subset[0, \infty)$ with $\mu_{n} \rightarrow 0$ as $n \rightarrow \infty$ such that

$$
F(T) \neq \emptyset, \quad \phi\left(p, T^{n} x\right) \leq\left(1+\mu_{n}\right) \phi(p, x), \quad \forall x \in C, \forall p \in F(T), \forall n \geq 1 .
$$

Remark 1.1 The class of relatively asymptotically nonexpansive mappings, which is an extension of the class of relatively nonexpansive mappings, was first introduced in [28].

Remark 1.2 The class of asymptotically quasi- $\phi$-nonexpansive mappings, which is an extension of the class of quasi- $\phi$-nonexpansive mappings, was considered in [29-31]. The class of quasi- $\phi$-nonexpansive mappings and the class of asymptotically quasi$\phi$-nonexpansive mappings are more general than the class of relatively nonexpansive mappings and the class of relatively asymptotically nonexpansive mappings. Quasi- $\phi$ nonexpansive mappings and asymptotically quasi- $\phi$-nonexpansive mappings do not require the restriction $F(T)=\widetilde{F}(T)$.

Recall that $T$ is said to be generalized asymptotically quasi- $\phi$-nonexpansive iff $F(T) \neq \emptyset$, and there exist two nonnegative sequences $\left\{\mu_{n}\right\} \subset[0, \infty)$ with $\mu_{n} \rightarrow 0$ and $\left\{\xi_{n}\right\} \subset[0, \infty)$ with $\xi_{n} \rightarrow 0$ as $n \rightarrow \infty$ such that

$$
\phi\left(p, T^{n} x\right) \leq\left(1+\mu_{n}\right) \phi(p, x)+\xi_{n}, \quad \forall x \in C, \forall p \in F(T), \forall n \geq 1 .
$$


Remark 1.3 The class of generalized asymptotically quasi- $\phi$-nonexpansive mappings [32] is a generalization of the class of generalized asymptotically quasi-nonexpansive mappings in the framework of Banach spaces which was studied by Agarwal et al. [33].

In this paper, we consider a projection algorithm for a common solution of a family of generalized asymptotically quasi- $\phi$-nonexpansive mappings and generalized mixed equilibrium problems. A strong convergence theorem is established in a Banach space. In order to prove our main results, we need the following lemmas.

Lemma 1.4 [21] Let $E$ be a uniformly convex Banach space, and let $r>0$. Then there exists a strictly increasing, continuous and convex function $g:[0,2 r] \rightarrow R$ such that $g(0)=0$ and

$$
\left\|\sum_{i=1}^{\infty}\left(\alpha_{i} x_{i}\right)\right\|^{2} \leq \sum_{i=1}^{\infty}\left(\alpha_{i}\left\|x_{i}\right\|^{2}\right)-\alpha_{i} \alpha_{j} g\left(\left\|x_{i}-x_{j}\right\|\right), \quad \forall i, j \in\{1,2, \ldots, N\}
$$

for all $x_{1}, x_{2}, \ldots, \in B_{r}=\{x \in E:\|x\| \leq r\}$ and $\alpha_{1}, \alpha_{2}, \ldots, \in[0,1]$ such that $\sum_{i=1}^{\infty} \alpha_{i}=1$.

Lemma 1.5 [1] Let $E$ be a reflexive, strictly convex and smooth Banach space, let $C$ be a nonempty closed convex subset of $E$ and $x \in E$. Then

$$
\phi\left(y, \Pi_{C} x\right)+\phi\left(\Pi_{C} x, x\right) \leq \phi(y, x), \quad \forall y \in C .
$$

Lemma 1.6 [1] Let $C$ be a nonempty closed convex subset of a smooth Banach space $E$ and $x \in E$. Then $x_{0}=\Pi_{C} x$ if and only if

$$
\left\langle x_{0}-y, J x-J x_{0}\right\rangle \geq 0, \quad \forall y \in C
$$

Lemma 1.7 [32] Let $E$ be a uniformly smooth and strictly convex Banach space which also enjoys the Kadec-Klee property, and let $C$ be a nonempty closed and convex subset of $E$. Let $T: C \rightarrow C$ be a generalized asymptotically quasi- $\phi$-nonexpansive mapping. Then $F(T)$ is closed and convex.

Lemma 1.8 [34] Let $C$ be a closed convex subset of a smooth, strictly convex and reflexive Banach space $E$. Let $A: C \rightarrow E^{*}$ be a continuous and monotone mapping, let $\varphi: C \rightarrow \mathbb{R}$ be convex and lower semi-continuous, and let $F$ be a bifunction from $C \times C$ to $\mathbb{R}$ satisfying (A1)-(A4). Let $r>0$ and $x \in E$. Then there exists $z \in C$ such that

$$
F(z, y)+\langle A z, y-z\rangle+\varphi(y)-\varphi(z)+\frac{1}{r}\langle y-z, J z-J x\rangle \geq 0, \quad \forall y \in C .
$$

Define a mapping $T_{r}: E \rightarrow C$ by

$$
T_{r} x=\left\{z \in C: F(z, y)+\langle A z, y-z\rangle+\varphi(y)-\varphi(z)+\frac{1}{r}\langle y-z, J z-J x\rangle \geq 0, \forall y \in C\right\} .
$$

Then the following conclusions hold:

(1) $T_{r}$ is a single-valued firmly nonexpansive-type mapping, i.e., for all $x, y \in E$,

$$
\left\langle T_{r} x-T_{r} y, J T_{r} x-J T_{r} y\right\rangle \leq\left\langle T_{r} x-T_{r} y, J x-J y\right\rangle ;
$$


(2) $F\left(T_{r}\right)=\operatorname{GMEP}(F, A, \varphi)$ is closed and convex;

(3) $T_{r}$ is quasi- $\phi$-nonexpansive;

(4) $\phi\left(q, T_{r} x\right)+\phi\left(T_{r} x, x\right) \leq \phi(q, x), \forall q \in F\left(T_{r}\right)$.

\section{Main results}

Theorem 2.1 Let E be a uniformly smooth and strictly convex Banach space which also enjoys the Kadec-Klee property, and let $C$ be a nonempty closed and convex subset of $E$. Let $\Delta$ be an index set and $N$ be an integer. Let $A_{j}: C \rightarrow E^{*}$ be a continuous and monotone mapping and $\varphi_{j}: C \rightarrow \mathbb{R}$ be a lower semi-continuous and convex function. Let $F_{j}$ be a bifunction from $C \times C$ to $\mathbb{R}$ satisfying (A1)-(A4) for every $j \in \Delta$. Let $T_{0}$ be an identity mapping, and let $T_{i}: C \rightarrow C$ be a generalized asymptotically quasi- $\phi$-nonexpansive mapping for every $1 \leq i \leq N$. Assume that $T_{i}$ is closed asymptotically regular on $C$ and $\Psi:=\bigcap_{i=1}^{N} F\left(T_{i}\right) \cap \bigcap_{j \in \Delta} \operatorname{GMEP}\left(F_{j}, A_{j}, \varphi_{j}\right)$ is nonempty and bounded. Let $\left\{x_{n}\right\}$ be a sequence generated in the following manner:

$$
\left\{\begin{array}{l}
x_{0} \in E, \text { chosen arbitrarily, } \\
C_{1, j}=C, \\
C_{1}=\bigcap_{j \in \Delta} C_{1, j}, \\
x_{1}=\Pi_{C_{1}} x_{0}, \\
y_{n}=J^{-1}\left(\sum_{i=0}^{N} \alpha_{n, i} J T_{i}^{n} x_{n}\right), \\
u_{n, j} \in C \text { such that } F_{j}\left(u_{n, j}, y\right)+\left\langle A_{j} u_{n, j}, y-u_{n, j}\right\rangle+\varphi_{j}(y)-\varphi_{j}\left(u_{n, j}\right) \\
\quad+\frac{1}{r_{n, j}}\left\langle y-u_{n, j}, J u_{n, j}-J y_{n}\right\rangle \geq 0, \quad \forall y \in C, \\
C_{n+1, j}=\left\{z \in C_{n}: \phi\left(z, u_{n, j}\right) \leq \phi\left(z, x_{n}\right)+\sum_{i=1}^{N} \mu_{n, i} M_{n}+N \xi_{n}\right\}, \\
C_{n+1}=\bigcap_{J \in \Delta} C_{n+1, J}, \\
x_{n+1}=\Pi_{C_{n+1}} x_{0},
\end{array}\right.
$$

where $\left\{\alpha_{n, i}\right\}$ is a real number sequence in $(0,1)$ for every $i \leq 1,\left\{r_{n, j}\right\}$ is a real number sequence in $[r, \infty)$, where $r$ is some positive real number, and $M_{n}=\sup \left\{\phi\left(z, x_{n}\right): z \in \Psi\right\}$. Assume that $\sum_{i=0}^{N} \alpha_{n, i}=1$ and $\liminf _{n \rightarrow \infty} \alpha_{n, 0} \alpha_{n, i}>0$ for every $1 \leq i \leq N$. Then the sequence $\left\{x_{n}\right\}$ converges strongly to $\Pi_{\Psi} x_{0}$, where $\Pi_{\Psi}$ is the generalized projection from E onto $\Psi$.

Proof The proof is split into five steps.

Step 1 . Show that the common solution set $\Psi$ is convex and closed.

This step is clear in view of Lemma 1.7 and Lemma 1.8.

Step 2. Show that the set $C_{n}$ is convex and closed.

To show Step 2, it suffices to show, for any fixed but arbitrary $i \in \Delta$, that $C_{n, i}$ is convex and closed. This can be proved by induction. It is clear that $C_{1, j}=C$ is convex and closed. Assume that $C_{m, j}$ is closed and convex for some $m \geq 1$. We next prove that $C_{m+1, j}$ is convex and closed. It is clear that $C_{m+1, j}$ is closed. We only prove they are convex. Indeed, $\forall x, y \in$ $C_{m+1, j}$, we find that $x, y \in C_{m, j}$, and

$$
\phi\left(x, u_{m, j}\right) \leq \phi\left(x, x_{m}\right)+\sum_{i=1}^{N} \mu_{n, i} M_{n}+N \xi_{n}
$$


and

$$
\phi\left(y, u_{m, j}\right) \leq \phi\left(y, x_{m}\right)+\sum_{i=1}^{N} \mu_{n, i} M_{n}+N \xi_{n}
$$

Notice that the above two inequalities are equivalent to the following inequalities, respectively:

$$
2\left\langle x, J x_{m}-J u_{m, j}\right\rangle \leq\left\|x_{m}\right\|^{2}-\left\|u_{m, j}\right\|^{2}+\sum_{i=1}^{N} \mu_{n, i} M_{n}+N \xi_{n}
$$

and

$$
2\left\langle y, J x_{m}-J u_{m, j}\right\rangle \leq\left\|x_{m}\right\|^{2}-\left\|u_{m, j}\right\|^{2}+\sum_{i=1}^{N} \mu_{n, i} M_{n}+N \xi_{n} .
$$

These imply that

$$
2\left\langle a x+(1-a) y, J x_{m}-J u_{m, j}\right\rangle \leq\left\|x_{m}\right\|^{2}-\left\|u_{m, j}\right\|^{2}+\sum_{i=1}^{N} \mu_{n, i} M_{n}+N \xi_{n}, \quad \forall a \in(0,1) .
$$

Since $C_{m, j}$ is convex, we see that $a x+(1-a) y \in C_{m, j}$. Notice that the above inequality is equivalent to

$$
\phi\left(a x+(1-a) y, u_{m, j}\right) \leq \phi\left(a x+(1-a) y, x_{m}\right)+\sum_{i=1}^{N} \mu_{n, i} M_{n}+N \xi_{n} .
$$

This proves that $C_{m+1, j}$ is convex. This proves that $C_{n}$ is closed and convex. This completes Step 2.

Step 3. Show that $\Psi \subset C_{n}$.

It suffices to claim that $\Psi \subset C_{n, j}$ for every $j \in \Delta$. Note that $\Psi \subset C_{1, j}=C$. Suppose that $\Psi \subset C_{m, j}$ for some $m$ and for every $j \in \Delta$. Then, for $\forall z \in \Psi \subset C_{m, j}$, we have

$$
\begin{aligned}
\phi\left(z, u_{m, j}\right)= & \phi\left(z, T_{r_{m, j}} y_{m}\right) \\
\leq & \phi\left(z, y_{m}\right) \\
= & \phi\left(z, J^{-1}\left(\alpha_{m, 0} J x_{m}+\sum_{i=1}^{N} \alpha_{m, i} J T_{i}^{m} x_{m}\right)\right) \\
= & \|z\|^{2}-2\left\langle z, \alpha_{m, 0} J x_{m}+\sum_{i=1}^{N} \alpha_{m, i} J T_{i}^{m} x_{m}\right\rangle+\left\|\alpha_{m, 0} J x_{m}+\sum_{i=1}^{N} \alpha_{m, i} J T_{i}^{m} x_{m}\right\|^{2} \\
\leq & \|z\|^{2}-2 \alpha_{m, 0}\left\langle z, J x_{m}\right\rangle-2 \sum_{i=1}^{N} \alpha_{m, i}\left\langle z, J T_{i}^{m} x_{m}\right\rangle \\
& +\alpha_{m, 0}\left\|x_{m}\right\|^{2}+\sum_{i=1}^{N} \alpha_{m, i}\left\|T_{i}^{m} x_{m}\right\|^{2}
\end{aligned}
$$




$$
\begin{aligned}
& =\alpha_{m, 0} \phi\left(z, x_{m}\right)+\sum_{i=1}^{N} \alpha_{m, i} \phi\left(z, T_{i}^{m} x_{m}\right) \\
& \leq \alpha_{m, 0} \phi\left(z, x_{m}\right)+\sum_{i=1}^{N} \alpha_{m, i} \phi\left(z, x_{m}\right)+\sum_{i=1}^{N} \alpha_{m, i} \mu_{m, i} \phi\left(z, x_{m}\right)+\sum_{i=1}^{N} \alpha_{m, i} \xi_{m} \\
& \leq \phi\left(z, x_{m}\right)+\sum_{i=1}^{N} \mu_{m, i} \phi\left(z, x_{m}\right)+\sum_{i=1}^{N} \alpha_{m, i} \xi_{m} \\
& \leq \phi\left(z, x_{m}\right)+\sum_{i=1}^{N} \mu_{m, i} M_{m}+\sum_{i=1}^{N} \alpha_{m, i} \xi_{m} \\
& \leq \phi\left(z, x_{m}\right)+\sum_{i=1}^{N} \mu_{m, i} M_{m}+N \xi_{m},
\end{aligned}
$$

which proves that $z \in C_{m+1, j}$. This completes Step 3.

Step 4. Show that $x_{n} \rightarrow p$, where $p \in \Psi$.

In view of Lemma 1.5 , we find that $\phi\left(x_{n}, x_{0}\right) \leq \phi\left(w, x_{0}\right)-\phi\left(w, x_{n}\right) \leq \phi\left(w, x_{0}\right)$ for $\forall w \in \Psi \subset$ $C_{n}$. This shows that the sequence $\phi\left(x_{n}, x_{0}\right)$ is bounded. It follows that $\left\{x_{n}\right\}$ is also bounded. Since the framework of the space is reflexive, we may, without loss of generality, assume that $x_{n} \rightarrow p$, where $p \in C_{n}$. Note that $\phi\left(x_{n}, x_{0}\right) \leq \phi\left(p, x_{0}\right)$. It follows that

$$
\phi\left(p, x_{0}\right) \leq \liminf _{n \rightarrow \infty} \phi\left(x_{n}, x_{0}\right) \leq \limsup _{n \rightarrow \infty} \phi\left(x_{n}, x_{0}\right) \leq \phi\left(p, x_{0}\right) .
$$

This gives that $\lim _{n \rightarrow \infty} \phi\left(x_{n}, x_{0}\right)=\phi\left(p, x_{0}\right)$. Hence, we have $\lim _{n \rightarrow \infty}\left\|x_{n}\right\|=\|p\|$. Since the space $E$ enjoys the Kadec-Klee property, we find that $x_{n} \rightarrow p$ as $n \rightarrow \infty$.

Now, we are in a position to show that $p \in \bigcap_{j \in \Delta} \operatorname{GMEP}\left(F_{j}, A_{j}, \varphi_{j}\right)$. By the construction of $C_{n}$, we have that $C_{n+1} \subset C_{n}$ and $x_{n+1}=\Pi_{C_{n+1}} x_{0} \in C_{n}$. It follows that

$$
\begin{aligned}
\phi\left(x_{n+1}, x_{n}\right) & =\phi\left(x_{n+1}, \Pi_{C_{n}} x_{0}\right) \\
& \leq \phi\left(x_{n+1}, x_{0}\right)-\phi\left(\Pi_{C_{n}} x_{0}, x_{0}\right) \\
& =\phi\left(x_{n+1}, x_{0}\right)-\phi\left(x_{n}, x_{0}\right) .
\end{aligned}
$$

Letting $n \rightarrow \infty$, we obtain that $\phi\left(x_{n+1}, x_{n}\right) \rightarrow 0$. In view of $x_{n+1} \in C_{n+1}$, we see that

$$
\phi\left(x_{n+1}, u_{n, j}\right) \leq \phi\left(x_{n+1}, x_{n}\right)+\sum_{i=1}^{N} \mu_{n, i} M_{n}+N \xi_{n} .
$$

We, therefore, obtain that $\lim _{n \rightarrow \infty} \phi\left(x_{n+1}, u_{n, j}\right)=0$. It follows that $\lim _{n \rightarrow \infty}\left\|u_{n, j}\right\|=\|p\|$. It follows that $\lim _{n \rightarrow \infty}\left\|J u_{n, j}\right\|=\|J p\|$. This implies that $\left\{J u_{n, j}\right\}$ is bounded. Note that $E$ is reflexive and $E^{*}$ is also reflexive. We may assume that $J u_{n, j} \rightarrow u^{*, j} \in E^{*}$. In view of the reflexivity of $E$, we see that $J(E)=E^{*}$. This shows that there exists $u^{j} \in E$ such that $J u^{j}=u^{* j}$. It follows that $\phi\left(x_{n+1}, u_{n}\right)=\left\|x_{n+1}\right\|^{2}-2\left\langle x_{n+1}, J u_{n}\right\rangle+\left\|J u_{n}\right\|^{2}$. Taking $\liminf _{n \rightarrow \infty}$ on the both 
sides of the equality above yields that

$$
\begin{aligned}
0 & \geq\|p\|^{2}-2\left\langle p, u^{*, j}\right\rangle+\left\|u^{*, j}\right\|^{2} \\
& =\|p\|^{2}-2\left\langle p, J u^{j}\right\rangle+\left\|J u^{j}\right\|^{2} \\
& =\|p\|^{2}-2\left\langle p, J u^{j}\right\rangle+\left\|u^{j}\right\|^{2} \\
& =\phi\left(p, u^{j}\right) .
\end{aligned}
$$

That is, $p=u^{j}$, which in turn implies that $J p=u^{*, j}$. It follows that $J u_{n, j} \rightarrow J p \in E^{*}$. Since $E^{*}$ enjoys the Kadec-Klee property, we obtain that $J u_{n, j}-J p \rightarrow 0$ as $n \rightarrow \infty$. Since $J^{-1}: E^{*} \rightarrow E$ is demicontinuous, it follows that $u_{n, j} \rightarrow p$. Since $E$ enjoys the Kadec-Klee property, we obtain that $u_{n, j} \rightarrow p$ as $n \rightarrow \infty$. Note that $\left\|x_{n}-u_{n, j}\right\| \leq\left\|x_{n}-p\right\|+\left\|p-u_{n, j}\right\|$. This gives that

$$
\lim _{n \rightarrow \infty}\left\|x_{n}-u_{n, j}\right\|=0
$$

Since $J$ is uniformly norm-to-norm continuous on any bounded sets, we have

$$
\lim _{n \rightarrow \infty}\left\|J x_{n}-J u_{n, j}\right\|=0
$$

Notice that

$$
\begin{aligned}
\phi\left(z, x_{n}\right)-\phi\left(z, u_{n, j}\right) & =\left\|x_{n}\right\|^{2}-\left\|u_{n, j}\right\|^{2}-2\left\langle z, J x_{n}-J u_{n, j}\right\rangle \\
& \leq\left\|x_{n}-u_{n, j}\right\|\left(\left\|x_{n}\right\|+\left\|u_{n, j}\right\|\right)+2\|z\|\left\|J x_{n}-J u_{n, j}\right\| .
\end{aligned}
$$

It follows from (2.2) and (2.3) that

$$
\lim _{n \rightarrow \infty} \phi\left(z, x_{n}\right)-\phi\left(z, u_{n, j}\right)=0
$$

From (2.1), we find that $\phi\left(z, y_{n}\right) \leq \phi\left(z, x_{n}\right)+\sum_{i=1}^{N} \mu_{n, j} M_{n}+N \xi_{n}$, where $z \in \Psi$. In view of $u_{n, j}=S_{r_{n, j}} y_{n}$, we find from Lemma 1.8 that

$$
\begin{aligned}
\phi\left(u_{n, j}, y_{n}\right) & =\phi\left(S_{r_{n, j}} y_{n}, y_{n}\right) \\
& \leq \phi\left(z, y_{n}\right)-\phi\left(z, S_{r_{n, j}} y_{n}\right) \\
& \leq \phi\left(z, x_{n}\right)-\phi\left(z, S_{r_{n, j}} y_{n}\right)+\sum_{i=1}^{N} \mu_{n, j} M_{n}+N \xi_{n} \\
& =\phi\left(z, x_{n}\right)-\phi\left(z, u_{n, j}\right)+\sum_{i=1}^{N} \mu_{n, j} M_{n}+N \xi_{n} .
\end{aligned}
$$

From (2.4), we obtain that

$$
\lim _{n \rightarrow \infty} \phi\left(u_{n, j}, y_{n}\right)=0
$$

This implies that $\left\|u_{n, j}\right\|-\left\|y_{n}\right\| \rightarrow 0$ as $n \rightarrow \infty$. Since $u_{n, j} \rightarrow p$ as $n \rightarrow \infty$, we arrive at $\lim _{n \rightarrow \infty}\left\|y_{n}\right\|=\|p\|$. It follows that $\lim _{n \rightarrow \infty}\left\|J y_{n}\right\|=\|J p\|$. Since $E^{*}$ is also reflexive, we may 
assume that $J y_{n} \rightarrow y^{*} \in E^{*}$. In view of $J(E)=E^{*}$, we see that there exists $y \in E$ such that $J y=y^{*}$. It follows that

$$
\phi\left(u_{n, j}, y_{n}\right)=\left\|u_{n, j}\right\|^{2}-2\left\langle u_{n, j}, J y_{n}\right\rangle+\left\|J y_{n}\right\|^{2} .
$$

Taking $\liminf _{n \rightarrow \infty}$ on the both sides of the equality above yields that $0 \geq \phi(p, y)$. That is, $p=y$, which in turn implies that $y^{*}=J p$. It follows that $J y_{n} \rightarrow J p \in E^{*}$. Since $E^{*}$ enjoys the Kadec-Klee property, we obtain that $J y_{n}-J p \rightarrow 0$ as $n \rightarrow \infty$. Note that $J^{-1}: E^{*} \rightarrow E$ is demicontinuous. It follows that $y_{n} \rightarrow p$. Since $E$ enjoys the Kadec-Klee property, we obtain that $y_{n} \rightarrow p$ as $n \rightarrow \infty$. Since $\left\|u_{n, j}-y_{n}\right\| \leq\left\|u_{n, j}-p\right\|+\left\|p-y_{n}\right\|$, we find that $\lim _{n \rightarrow \infty} \| u_{n, i}-$ $y_{n} \|=0$. Since $J$ is uniformly norm-to-norm continuous on any bounded sets, we have $\lim _{n \rightarrow \infty}\left\|J u_{n, j}-J y_{n}\right\|=0$. From the assumption $r_{n, i} \geq r$, we see that $\lim _{n \rightarrow \infty} \frac{\left\|J_{n, j}-J y_{n}\right\|}{r_{n, j}}=0$. Notice that

$$
f_{j}\left(u_{n, j}, y\right)+\frac{1}{r_{n, j}}\left\langle y-u_{n, j}, J u_{n, j}-J y_{n}\right\rangle \geq 0, \quad \forall y \in C,
$$

where $f_{j}\left(u_{n, j}, y\right)=F_{j}\left(u_{n, j}, y\right)+\left\langle A_{j} u_{n, j}, y-u_{n, j}\right\rangle+\varphi_{j}(y)-\varphi_{j}\left(u_{n, j}\right)$. From (A2), we find that

$$
\left\|y-u_{n, j}\right\| \frac{\left\|J u_{n, j}-J y_{n}\right\|}{r_{n, j}} \geq \frac{1}{r_{n, j}}\left\langle y-u_{n, j}, J u_{n, j}-J y_{n}\right\rangle \geq f_{j}\left(y, u_{n, j}\right), \quad \forall y \in C .
$$

Taking the limit as $n \rightarrow \infty$, we find that $f_{j}(y, p) \leq 0, \forall y \in C$. For $0<t_{j}<1$ and $y \in C$, define $y_{t_{j}}=t_{j} y+\left(1-t_{j}\right) p$. It follows that $y_{t, j} \in C$, which yields that $f_{j}\left(y_{t, j}, p\right) \leq 0$. It follows from conditions (A1) and (A4) that $0=f_{j}\left(y_{t, j}, y_{t, j}\right) \leq t_{j} f_{j}\left(y_{t, j}, y\right)+\left(1-t_{j}\right) f_{j}\left(y_{t, j}, p\right) \leq t_{j} f_{j}\left(y_{t, j}, y\right)$. This yields that $f_{j}\left(y_{t, j}, y\right) \geq 0$. Letting $t_{j} \downarrow 0$, we find from condition (A3) that $f_{j}(p, y) \geq 0, \forall y \in C$. This implies that $p \in E P\left(f_{j}\right)=\operatorname{GMEP}\left(F_{j}, A_{j}, \varphi_{j}\right)$ for every $j \in \Delta$.

Next, we state $p \in \bigcap_{i=1}^{N} F\left(T_{i}\right)$. Since $E$ is uniformly smooth, we know that $E^{*}$ is uniformly convex. It follows from Lemma 1.4 that

$$
\begin{aligned}
\phi\left(z, u_{n, j}\right)= & \phi\left(z, S_{r_{n, j}} y_{n}\right) \\
\leq & \phi\left(z, y_{n}\right) \\
= & \phi\left(z, J^{-1}\left(\alpha_{n, 0} J x_{n}+\sum_{i=1}^{N} \alpha_{n, i} J T_{i}^{n} x_{n}\right)\right) \\
= & \|z\|^{2}-2\left\langle z, \alpha_{n, 0} J x_{n}+\sum_{i=1}^{N} \alpha_{n, j} J T_{i}^{n} x_{n}\right\rangle+\left\|\alpha_{n, 0} J x_{n}+\sum_{i=1}^{N} \alpha_{n, i} J T_{i}^{n} x_{n}\right\|^{2} \\
\leq & \|z\|^{2}-2 \alpha_{n, 0}\left\langle z, J x_{n}\right\rangle-2 \sum_{i=1}^{N} \alpha_{n, i}\left\langle z, J T_{i}^{n} x_{n}\right\rangle \\
& +\alpha_{n, 0}\left\|x_{n}\right\|^{2}+\sum_{i=1}^{N} \alpha_{n, i}\left\|T_{i}^{n} x_{n}\right\|^{2}-\alpha_{n, 0}\left(1-\alpha_{n, i}\right) g\left(\left\|J x_{n}-J T_{i}^{n} x_{n}\right\|\right) \\
= & \alpha_{n, 0} \phi\left(z, x_{m}\right)+\sum_{i=1}^{N} \alpha_{n, i} \phi\left(z, T_{i}^{n} x_{n}\right)-\alpha_{n, 0}\left(1-\alpha_{n, i}\right) g\left(\left\|J x_{n}-J T_{i}^{n} x_{n}\right\|\right) \\
\leq & \alpha_{n, 0} \phi\left(z, x_{m}\right)+\sum_{i=1}^{N} \alpha_{n, i} \phi\left(z, x_{m}\right)+\sum_{i=1}^{N} \alpha_{n, i} \mu_{n, i} \phi\left(z, x_{n}\right)+\sum_{i=1}^{N} \alpha_{n, i} \xi_{n}
\end{aligned}
$$




$$
\begin{aligned}
& -\alpha_{n, 0}\left(1-\alpha_{n, i}\right) g\left(\left\|J x_{n}-J T_{i}^{n} x_{n}\right\|\right) \\
\leq & \phi\left(z, x_{n}\right)+\sum_{i=1}^{N} \mu_{n, i} \phi\left(z, x_{m}\right)+\sum_{i=1}^{N} \alpha_{n, i} \xi_{n} \\
& -\alpha_{n, 0}\left(1-\alpha_{n, i}\right) g\left(\left\|J x_{n}-J T_{i}^{n} x_{n}\right\|\right) \\
\leq & \phi\left(z, x_{n}\right)+\sum_{i=1}^{N} \mu_{n, i} M_{n}+N \xi_{n}-\alpha_{n, 0}\left(1-\alpha_{n, i}\right) g\left(\left\|J x_{n}-J T_{i}^{n} x_{n}\right\|\right) .
\end{aligned}
$$

This yields that

$$
\alpha_{n, 0}\left(1-\alpha_{n, i}\right) g\left(\left\|J x_{n}-J T_{i}^{n} x_{n}\right\|\right) \leq \phi\left(z, x_{n}\right)-\phi\left(z, u_{n, j}\right)+\sum_{i=1}^{N} \mu_{n, i} M_{n}+N \xi_{n}
$$

In view of $\liminf _{n \rightarrow \infty} \alpha_{n, 0}\left(1-\alpha_{n, i}\right)>0$, we see from (2.4) that $\lim _{n \rightarrow \infty} g\left(\left\|J x_{n}-J T_{i}^{n} x_{n}\right\|\right)=0$ It follows from the property of $g$ that

$$
\lim _{n \rightarrow \infty}\left\|J x_{n}-J T_{i}^{n} x_{n}\right\|=0
$$

Since $x_{n} \rightarrow p$ as $n \rightarrow \infty$ and $J: E \rightarrow E^{*}$ is demicontinuous, we obtain that $J x_{n} \rightarrow J p \in E^{*}$. Note that $\left|\left\|J x_{n}\right\|-\|J p\|\right|=\left|\left\|x_{n}\right\|-\|p\|\right| \leq\left\|x_{n}-p\right\|$. This implies that $\left\|J x_{n}\right\| \rightarrow\|J p\|$ as $n \rightarrow \infty$. Since $E^{*}$ enjoys the Kadec-Klee property, we see that

$$
\lim _{n \rightarrow \infty}\left\|J x_{n}-J p\right\|=0
$$

On the other hand, we have $\left\|J T_{i}^{n} x_{n}-J p\right\| \leq\left\|J T_{i}^{n} x_{n}-J x_{n}\right\|+\left\|J x_{n}-J p\right\|$. Combining (2.5) with (2.6), one obtains that $\lim _{n \rightarrow \infty}\left\|J T_{i}^{n} x_{n}-J p\right\|=0$. Since $J^{-1}: E^{*} \rightarrow E$ is demicontinuous, one sees that $T_{i}^{n} x_{n} \rightarrow p$. Notice that $\left|\left\|T_{i}^{n} x_{n}\right\|-\|p\|\right| \leq\left\|J T_{i}^{n} x_{n}-J p\right\|$. This yields that $\lim _{n \rightarrow \infty}\left\|T_{i}^{n} x_{n}\right\|=\|p\|$. Since the space $E$ enjoys the Kadec-Klee property, we obtain that $\lim _{n \rightarrow \infty}\left\|T_{i}^{n} x_{n}-p\right\|=0$. Note that $\left\|T^{n+1} x_{n}-p\right\| \leq\left\|T^{n+1} x_{n}-T^{n} x_{n}\right\|+\left\|T^{n} x_{n}-p\right\|$. Since $T$ is asymptotically regular, we find that $\lim _{n \rightarrow \infty}\left\|T_{i}^{n+1} x_{n}-p\right\|=0$. That is, $T_{i} T_{i}^{n} x_{n}-p \rightarrow 0$ as $n \rightarrow \infty$. It follows from the closedness of $T_{i}$ that $T_{i} p=p$ for every $i \in\{1,2, \ldots, N\}$. This completes Step 4.

Step 5. Show that $p=\Pi_{\Psi} x_{0}$.

Since $x_{n}=\Pi_{C_{n}} x_{0}$, we see that

$$
\left\langle x_{n}-z, J x_{0}-J x_{n}\right\rangle \geq 0, \quad \forall z \in C_{n} .
$$

Since $\Psi \subset C_{n}$, we find that

$$
\left\langle x_{n}-w, J x_{0}-J x_{n}\right\rangle \geq 0, \quad \forall w \in \Psi
$$

Letting $n \rightarrow \infty$, we arrive at

$$
\left\langle p-w, J x_{0}-J p\right\rangle \geq 0, \quad \forall w \in \Psi
$$

From Lemma 1.6, we can immediately obtain that $p=\Pi_{\Psi} x_{0}$. This completes the proof. 
Remark 2.2 Theorem 2.1 mainly improves the corresponding results in Kim [20], Yang et al. [21], Hao [23], Qin et al. [31], Qin et al. [35].

Remark 2.3 The framework of the space in Theorem 2.1 can be applicable to $L^{p}, p \geq 1$.

If $N=2$ and $\Delta=\{1\}$, then Theorem 2.1 is reduced to the following.

Corollary 2.4 Let E be a uniformly smooth and strictly convex Banach space which also enjoys the Kadec-Klee property, and let $C$ be a nonempty closed and convex subset of E. Let $F$ be a bifunction from $C \times C$ to $\mathbb{R}$ satisfying (A1)-(A4). Let $T_{i}: C \rightarrow C$ be a generalized asymptotically quasi- $\phi$-nonexpansive mapping for every $i \in\{1,2\}$. Assume that each $T_{i}$ is closed asymptotically regular on $C$ and $F\left(T_{1}\right) \cap F\left(T_{2}\right) \cap E P(F)$ is nonempty and bounded. Let $\left\{x_{n}\right\}$ be a sequence generated in the following manner:

$$
\left\{\begin{array}{l}
x_{0} \in E, \text { chosen arbitrarily, } \\
C_{1}=C, \\
x_{1}=\Pi_{C_{1} x_{0}}, \\
y_{n}=J^{-1}\left(\alpha_{n, 0} J x_{n}+\alpha_{n, 1} J T_{1}^{n} x_{n}+\alpha_{n, 2} J T_{2}^{n} x_{n}\right), \\
u_{n} \in C \text { such that } F\left(u_{n}, y\right)+\frac{1}{r_{n}}\left\langle y-u_{n}, J u_{n}-J y_{n}\right\rangle \geq 0, \quad \forall y \in C, \\
C_{n+1}=\left\{z \in C_{n}: \phi\left(z, u_{n}\right) \leq \phi\left(z, x_{n}\right)+\left(\mu_{n, 1}+\mu_{n, 2}\right) M_{n}+2 \xi_{n}\right\}, \\
x_{n+1}=\Pi_{C_{n+1}} x_{0},
\end{array}\right.
$$

where $\left\{\alpha_{n, 0}\right\},\left\{\alpha_{n, 1}\right\}$, and $\left\{\alpha_{n, 2}\right\}$ are real number sequences in $(0,1),\left\{r_{n}\right\}$ is a real number sequence in $[r, \infty)$, where $r$ is some positive real number, and $M_{n}=\sup \left\{\phi\left(z, x_{n}\right): z \in F\left(T_{1}\right) \cap\right.$ $\left.F\left(T_{2}\right) \cap E F(F)\right\}$. Assume that $\sum_{i=0}^{2} \alpha_{n, i}=1$ and $\liminf _{n \rightarrow \infty} \alpha_{n, 0} \alpha_{n, i}>0$. Then the sequence $\left\{x_{n}\right\}$ converges strongly to $\Pi_{F\left(T_{1}\right) \cap F\left(T_{2}\right) \cap E P(F)} x_{0}$, where $\Pi_{F\left(T_{1}\right) \cap F\left(T_{2}\right) \cap E F(F)}$ is the generalized projection from $E$ onto $F\left(T_{1}\right) \cap F\left(T_{2}\right) \cap E P(F)$.

Remark 2.5 Corollary 2.4 mainly improves the corresponding results in Qin et al. [31]. To be more clear, the mapping is extended from quasi- $\phi$-nonexpansive mappings to generalized asymptotically quasi- $\phi$-nonexpansive mappings and the framework of spaces is extended from a uniformly smooth and uniformly convex Banach space to a uniformly smooth and strictly convex Banach space.

\footnotetext{
Competing interests

The authors declare that they have no competing interests.
}

Authors' contributions

Both authors contributed equally to this manuscript. Both authors read and approved the final manuscript.

\section{Author details}

${ }^{1}$ School of Business and Administration, Henan University, Kaifeng, 475000, China. ${ }^{2}$ Kaifeng Vocational College of Culture and Arts, Kaifeng, 475000, China.

\section{Acknowledgements}

The authors are grateful to the reviewers' useful suggestions which improved the contents of the article. 


\section{References}

1. Alber, Yl: Metric and generalized projection operators in Banach spaces: properties and applications. In: Kartsatos, AG (ed.) Theory and Applications of Nonlinear Operators of Accretive and Monotone Type. Dekker, New York (1996)

2. Cho, SY, Kang, SM: Approximation of common solutions of variational inequalities via strict pseudocontractions. Acta Math. Sci. 32, 1607-1618 (2012)

3. Shen, J, Pang, LP: An approximate bundle method for solving variational inequalities. Commun. Optim. Theory 1 , $1-18$ (2012)

4. Noor, MA, Noor, Kl, Waseem, M: Decomposition method for solving system of linear equations. Eng. Math. Lett. 2 , 34-41 (2013)

5. Qin, X, Cho, SY, Kang, SM: Iterative algorithms for variational inequality and equilibrium problems with applications. J. Glob. Optim. 48, 423-445 (2010)

6. Osu, BO, Solomon, OU: A stochastic algorithm for the valuation of financial derivatives using the hyperbolic distributional variates. Math. Finance Lett. 1, 43-56 (2012)

7. Cho, SY, Kang, SM: Approximation of fixed points of pseudocontraction semigroups based on a viscosity iterative process. Appl. Math. Lett. 24, 224-228 (2011)

8. Wang, ZM, Lou, W: A new iterative algorithm of common solutions to quasi-variational inclusion and fixed point problems. J. Math. Comput. Sci. 3, 57-72 (2013)

9. Zegeye, $\mathrm{H}$, Shahzad, N: Strong convergence theorem for a common point of solution of variational inequality and fixed point problem. Adv. Fixed Point Theory 2, 374-397 (2012)

10. Qin, X, Cho, SY, Kang, SM: An extragradient-type method for generalized equilibrium problems involving strictly pseudocontractive mappings. J. Glob. Optim. 49, 679-693 (2011)

11. He, RH: Coincidence theorem and existence theorems of solutions for a system of Ky Fan type minimax inequalities in FC-spaces. Adv. Fixed Point Theory 2, 47-57 (2012)

12. Cho, SY, Li, W, Kang, SM: Convergence analysis of an iterative algorithm for monotone operators. J. Inequal. Appl. 2013, Article ID 199 (2013)

13. Al-Bayati, AY, Al-Kawaz, RZ: A new hybrid WC-FR conjugate gradient-algorithm with modified secant condition for unconstrained optimization. J. Math. Comput. Sci. 2, 937-966 (2012)

14. Tanaka, Y: A constructive version of Ky Fan's coincidence theorem. J. Math. Comput. Sci. 2, 926-936 (2012)

15. Park, S: A review of the KKM theory on $\phi_{A}$-space or GFC-spaces. Adv. Fixed Point Theory 3, 355-382 (2013)

16. Cho, SY, Qin, X, Kang, SM: Iterative processes for common fixed points of two different families of mappings with applications. J. Glob. Optim. 57, 1429-1446 (2013)

17. Wang, G, Sun, S: Hybrid projection algorithms for fixed point and equilibrium problems in a Banach space. Adv. Fixed Point Theory 3, 578-594 (2013)

18. LV, S, Wu, C: Convergence of iterative algorithms for a generalized variational inequality and a nonexpansive mapping. Eng. Math. Lett. 1, 44-57 (2012)

19. Chen, JH: Iterations for equilibrium and fixed point problems. J. Nonlinear Funct. Anal. 2013, Article ID 4 (2013)

20. Kim, JK: Strong convergence theorems by hybrid projection methods for equilibrium problems and fixed point problems of the asymptotically quasi- $\phi$-nonexpansive mappings. Fixed Point Theory Appl. 2011, Article ID 10 (2011)

21. Yang, L, Zhao, F, Kim, JK: Hybrid projection method for generalized mixed equilibrium problem and fixed point problem of infinite family of asymptotically quasi- $\phi$-nonexpansive mappings in Banach spaces. Appl. Math. Comput. $218,6072-6082(2012)$

22. Cho, SY, Kang, SM: Zero point theorems for $m$-accretive operators in a Banach space. Fixed Point Theory 13, 49-58 (2012)

23. Hao, Y: On generalized quasi- $\phi$-nonexpansive mappings and their projection algorithms. Fixed Point Theory Appl. 2013, Article ID 204 (2013)

24. Lions, PL, Mercier, B: Splitting algorithms for the sum of two nonlinear operators. SIAM J. Numer. Anal. 16, 964-979 (1979)

25. Kang, SM, Cho, SY, Liu, Z: Convergence of iterative sequences for generalized equilibrium problems involving inverse-strongly monotone mappings. J. Inequal. Appl. 2010, Article ID 827082 (2010)

26. Wu, C: Wiener-Hopf equations methods for generalized variational inequalities. J. Nonlinear Funct. Anal. 2013, Article ID 3 (2013)

27. Wu, C: Mann iteration for zero theorems of accretive operators. J. Fixed Point Theory 2013, 3 (2013)

28. Agarwal, RP, Cho, YJ, Qin, X: Generalized projection algorithms for nonlinear operators. Numer. Funct. Anal. Optim. 28, 1197-1215 (2007)

29. Qin, X, Cho, SY, Kang, SM: On hybrid projection methods for asymptotically quasi- $\boldsymbol{\phi}$-nonexpansive mappings. Appl. Math. Comput. 215, 3874-3883 (2010)

30. Zhou, H, Gao, G, Tan, B: Convergence theorems of a modified hybrid algorithm for a family of quasi- $\phi$-asymptotically nonexpansive mappings. J. Appl. Math. Comput. 32, 453-464 (2010)

31. Qin, X, Cho, YJ, Kang, SM: Convergence theorems of common elements for equilibrium problems and fixed point problems in Banach spaces. J. Comput. Appl. Math. 225, 20-30 (2009)

32. Qin, X, Agarwal, RP, Cho, SY, Kang, SM: Convergence of algorithms for fixed points of generalized asymptotically quasi- $\phi$-nonexpansive mappings with applications. Fixed Point Theory Appl. 2012, Article ID 58 (2012)

33. Agarwal, RP, Qin, X, Kang, SM: An implicit iterative algorithm with errors for two families of generalized asymptotically nonexpansive mappings. Fixed Point Theory Appl. 2011, Article ID 58 (2011)

34. Chang, SS, Kim, JK, Wang, XR: Modified block iterative algorithm for solving convex feasibility problems in Banach spaces. J. Inequal. Appl. 2010, Article ID 869684 (2010)

35. Qin, $X$, Cho, SY, Kang, SM: Strong convergence of shrinking projection methods for quasi- $\phi$-nonexpansive mappings and equilibrium problems. J. Comput. Appl. Math. 234, 750-760 (2010)

10.1186/1687-1812-2013-318

Cite this article as: Wu and Sun: A monotone projection algorithm for fixed points of nonlinear operators. Fixed Point Theory and Applications 2013, 2013:318 